OPEN ACCESS

Edited by:

Jan Novak,

University of Alabama at Birmingham,

United States

Reviewed by:

Baidong Hou,

Institute of Biophysics (CAS), China

Ronald B. Corley,

Boston University, United States

Mariah Hassert,

The University of lowa, United States

*Correspondence:

Adam T. Waickman waickmaa@upstate.edu

Specialty section:

This article was submitted to B Cell Biology,

a section of the journal

Frontiers in Immunology

Received: 15 September 2021 Accepted: 08 November 2021 Published: 24 November 2021

Citation:

Wegman $A D$, Fang $H$, Rothman $A L$, Thomas SJ, Endy TP, McCracken MK, Currier JR, Friberg H, Gromowski GD and Waickman AT (2021) Monomeric IgA Antagonizes IgG-Mediated Enhancement of DENV Infection.

Front. Immunol. 12:777672. doi: 10.3389/fimmu.2021.777672

\section{Monomeric IgA Antagonizes IgG-Mediated Enhancement of DENV Infection}

\author{
Adam D. Wegman ${ }^{1}$, Hengsheng Fang ${ }^{1}$, Alan L. Rothman ${ }^{2}$, Stephen J. Thomas ${ }^{1,3}$, \\ Timothy P. Endy ${ }^{1}$, Michael K. McCracken ${ }^{4}$, Jeffrey R. Currier ${ }^{4}$, Heather Friberg ${ }^{4}$, \\ Gregory D. Gromowski ${ }^{4}$ and Adam T. Waickman ${ }^{1,3 *}$
}

\begin{abstract}
${ }^{1}$ Department of Microbiology and Immunology, State University of New York Upstate Medical University, Syracuse, NY, United States, ${ }^{2}$ Department of Cell and Molecular Biology, Institute for Immunology and Informatics, University of Rhode Island, Providence, RI, United States, ${ }^{3}$ Institute for Global Health and Translational Sciences, State University of New York Upstate Medical University, Syracuse, NY, United States, ${ }^{4}$ Viral Diseases Branch, Walter Reed Army Institute of Research, Silver Spring, MD, United States
\end{abstract}

Dengue virus (DENV) is a prevalent human pathogen, infecting approximately 400 million individuals per year and causing symptomatic disease in approximately 100 million. A distinct feature of dengue is the increased risk for severe disease in some individuals with preexisting DENV-specific immunity. One proposed mechanism for this phenomenon is antibody-dependent enhancement (ADE), in which poorly-neutralizing IgG antibodies from a prior infection opsonize DENV to increase infection of $F_{C}$ gamma receptorbearing cells. While IgM and IgG are the most commonly studied DENV-reactive antibody isotypes, our group and others have described the induction of DENV-specific serum IgA responses during dengue. We hypothesized that monomeric IgA would be able to neutralize DENV without the possibility of ADE. To test this, we synthesized IgG and IgA versions of two different DENV-reactive monoclonal antibodies. We demonstrate that isotype-switching does not affect the antigen binding and neutralization properties of the two mAbs. We show that DENV-reactive IgG, but not IgA, mediates ADE in $F_{c}$ gamma receptor-positive K562 cells. Furthermore, we show that IgA potently antagonizes the ADE activity of IgG. These results suggest that levels of DENV-reactive IgA induced by DENV infection might regulate the overall IgG mediated ADE activity of DENV-immune plasma in vivo, and may serve as a predictor of disease risk.

Keywords: Dengue, DENV, antibody dependent enhancement, IgA, ADE

\section{INTRODUCTION}

Dengue virus (DENV) is one of the most widespread vector-borne viral pathogens in the world. Consisting of four antigenically and genetically distinct serotypes (DENV-1,-2, -3, and -4), DENV is transmitted primarily by the tropical and subtropical mosquitoes Aedes aegypti and A. albopictus (1, 2). DENV and its mosquito vectors can currently be found across Central and South America, South and South-East Asia, the Western Pacific, and sub-Saharan Africa, meaning 40\% of the world's population is currently at risk of exposure and infection (1-3). Consequently, an estimated 400 
million DENV infections are thought to occur every year, resulting in 100 million clinically apparent infections (2). Approximately 500,000 cases per year progress to severe dengue-characterized by thrombocytopenia, vascular leakage and hemorrhage-resulting in nearly 20,000 deaths (4-7).

A distinct epidemiological feature of dengue as compared to other flaviviral diseases is the increased risk for severe disease upon heterologous secondary infection (8). While the risk factors associated with developing severe dengue upon secondary DENV exposure are complex and incompletely understood, the leading mechanistic explanation for this phenomenon is a process known as antibody-dependent enhancement $(\operatorname{ADE})(9,10)$. ADE is thought to occur when poorly-neutralizing or sub-neutralizing concentrations of DENV-reactive IgG opsonize DENV and facilitate its entry into permissive $\mathrm{F}_{\mathrm{c}} \gamma \mathrm{R}$-bearing cells (11). Various lines of evidence support the association of ADE with severe dengue, including increased incidence of severe dengue in infants born to dengue-immune mothers (12-14); increased viremia in interferon receptor-deficient mice or non-human primates passively immunized with anti-DENV antibodies (15, 16); increased incidence of severe dengue during the second of sequential heterologous DENV outbreaks; and in patients with a narrow range of preexisting anti-DENV antibody titers $(17,18)$. Furthermore, in-vitro assessments of serum ADE activity in DENVprimed non-human primates have been shown to correlate with viral titers following heterologous attenuated DENV infection (19).

The increased risk of severe dengue upon secondary heterologous infection also presents a challenge to vaccine development as incomplete or waning vaccine-elicited immunity may place recipients at an increased risk of developing severe dengue should they be exposed following vaccination (20). This is most significantly highlighted by the revelation that the only currently US FDA licensed DENV vaccine (Dengvaxia ${ }^{\circledR}$ ) fails to protect previously DENV naïve individuals from infection, and can increase the risk of hospitalization with virologically confirmed dengue (21-23). Accordingly, understanding the subtleties of both natural and vaccine-elicited DENV humoral immunity is critical for further our understanding of disease risk and infection-associated immunopathogenesis.

To date, the literature on dengue serology has overwhelmingly focused on the contribution of immunoglobulin isotypes IgM and IgG to functional dengue immunity and infection-associated immunopathogenesis. During both primary and secondary DENV infection, these isotype antibodies follow a highly predictable pattern of induction, with an IgM response preceding the rise of DENV-reactive IgG, and DENV-reactive IgG reaching significantly higher titers during secondary infection $(24,25)$. These characteristics, as well as the assumed importance of IgG-mediated ADE, have left the role of other serum antibody isotypes relatively unexamined. Notably, this includes IgA, the second most prevalent antibody isotype in serum and one that has been suggested to play a unique and non-redundant role in many viral infections (26). Most work on DENV-reactive serum IgA has focused on its potential as a diagnostic tool (27), with a small body of literature examining DENV-reactive serum IgA as a possible correlate of severe disease (28-31).
Our group and others recently reported that IgA was the dominant isotype-switched antibody expressed by circulating plasmablasts during acute primary DENV infection $(32,33)$. IgA-expressing plasmablasts were also observed in secondary dengue, but constituted a smaller fraction of the total infectionelicited immune response $(32,33)$. Importantly, the IgA antibodies expressed by these plasmablasts exhibited comparable DENV-binding and DENV-neutralization activity to IgG antibodies derived from contemporaneous samples (32). Given the milder symptoms and lower viral burden typically associated with primary dengue relative to secondary dengue, we hypothesized that DENV-reactive IgA may play some role in limiting DENV propagation and potentially the immunemediated enhancement of disease.

To test this hypothesis, we isotype-switched pairs of monoclonal antibodies to show that conversion of IgG to IgA does not impact the ability of a monoclonal antibody to bind whole DENV virions or to neutralize DC-SIGN-dependent DENV infection of a susceptible cell line. However, while DENV-reactive IgG antibodies exhibited potent infection-enhancing activity in in vitro $\mathrm{ADE}$ assays, we observed that $\mathrm{DENV}$-reactive $\operatorname{IgA}$ is incapable of mediating ADE. Additionally, we observed that adding DENV-reactive monoclonal IgA to either an enhancing concentration of monoclonal IgG or to an enhancing dilution of dengue-immune plasma antagonizes $\mathrm{ADE}$ in a dose-dependent fashion. These results shed new light on the role of the IgA component of the humoral response to DENV, and suggest a new avenue of prophylactic and therapeutic approaches to disease.

\section{RESULTS}

\section{DENV Binding and Neutralizing Are Unaffected by Antibody $F_{c}$ Isotype}

To assess the potential contribution of DENV-reactive IgA to a functional anti-DENV humoral immune response, we synthesized two pairs of previously described DENV-reactive monoclonal antibodies with either an IgG or an IgA Fc domain (Figure 1A). Both $\mathrm{mAbs}$ selected for this analysis were previously determined to bind the fusion loop of the DENV E protein and to react with all 4 DENV serotypes (32). However, VDB33 was initially identified as an IgG clone, while VDB50 was discovered as an IgA clone (Table 1). This cross-conversion strategy was chosen so as to determine if the native $\mathrm{F}_{\mathrm{c}}$ configuration of a given antibody influenced its functionality as either an IgG or IgA protein product.

The DENV-binding capacity of the IgG and IgA versions of VDB33 and VDB50 was initially assessed with a DENV virioncapture ELISA. For this analysis, DENV-3 was chosen as the prototypic DENV serotype as previous work demonstrated that the IgG versions of both VDB33 and VDB50 exhibited significant DENV-3 reactivity (32). Consistent with previously published reports, both VDB33 and VDB50 exhibited potent DENV-3 binding activity with VDB33 demonstrating 200 fold higher affinity for DENV-3 than VDB50 (Figure 1B and Table 2). However, the DENV-binding capacity of the two mAbs was not impacted by their conversion to either an IgG or IgA format 
A

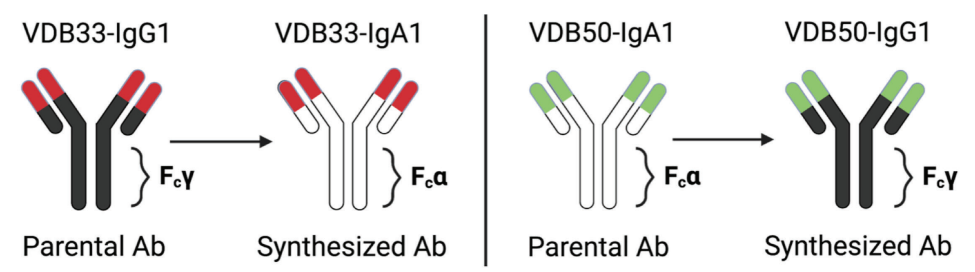

B

DENV-3 virion binding (ELISA)
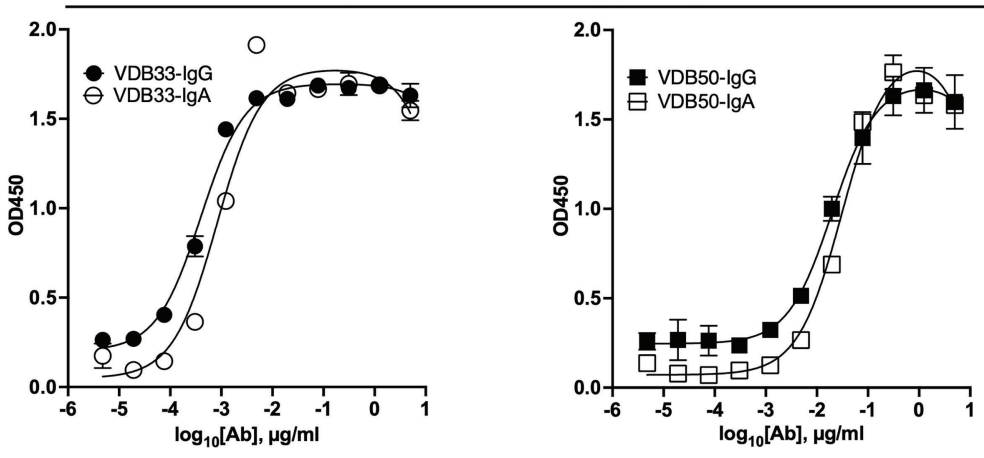

C

DENV-3 neutralization (FlowNT)
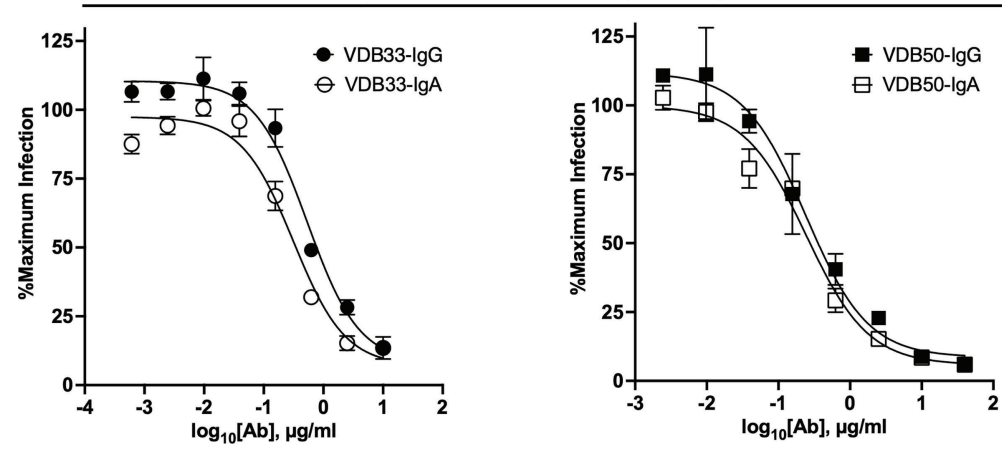

FIGURE 1 | Isotype conversion scheme, DENV binding, and DENV neutralization capacity of VDB33 and VDB50 mAbs. (A) Schematic of isotype conversion of VDB33 and VDB50 from respective parental isotypes, indicating conservation of antigen-binding domains and alteration of $F_{C}$ domains. (B) DENV-3 binding capability of VDB33-IgG, VDB33-IgA, VDB50-IgG, and VDB50-IgA measured by DENV virus-capture ELISA. (C) DENV-3 neutralization capability of VDB33-IgG, VDB33-IgA, VDB50-IgG, and VDB50-IgA as assessed by FlowNT. Neutralization data are presented as a percent of the positive (no neutralizing mAb) control for each replicate. Error bars +/- SEM. Data are representative of at least 4 independent experiments.

TABLE 1 | Sequence information of DENV-reactive monoclonal antibodies.

\begin{tabular}{|c|c|c|}
\hline Clone name & VDB33 & VDB50 \\
\hline Infecting Serotype & DENV-3 & DENV-1 \\
\hline Primary/Secondary & Secondary & Primary \\
\hline $\mathbf{H}_{\mathrm{c}} \mathbf{V}$ & IGHV4-39*01 & IGHV3-23*03 \\
\hline$H_{c} \mathbf{D}$ & IGHD1-701 & IGHD5-24*01 \\
\hline $\mathbf{H}_{\mathrm{c}} \mathbf{J}$ & IGHJ502 & IGHJ4*02 \\
\hline $\mathrm{L}_{\mathrm{C}} \mathrm{V}$ & IGLV3-21*03 & IGLV1-40*01 \\
\hline $\mathbf{L}_{c} \mathbf{J}$ & IGLJ3*02 & IGLJ3*02 \\
\hline $\mathrm{L}_{\mathrm{C}}$ Total SHM & 14 & 8 \\
\hline Target residues & W101, G106, L107, F108 & G100, W101, F108 \\
\hline E protein epitope & Fusion loop & Fusion loop \\
\hline
\end{tabular}


TABLE 2 | Functional characteristics isotype-switched monoclonal antibodies.

\begin{tabular}{|c|c|c|c|c|}
\hline Clone name & VDB33 IgG & VDB33 IgA & VDB50 IgG & VDB50 $\lg A$ \\
\hline Kd (ng/mL) & 0.3962 & 0.8296 & 19.92 & 31.12 \\
\hline IC50 (ug/mL) & 0.5339 & 0.3139 & 0.2391 & 0.2439 \\
\hline
\end{tabular}

(Figure 1B and Table 2). Furthermore, this cross-conversion of VDB33 and VDB50 to either an IgG or IgA format minimally impacted the DENV-3 neutralization activity of the clones when assessed using a flow cytometry-based neutralization assay (Figure 1C and Table 2). These results indicate that both IgG and IgA isotype antibodies are equally capable of binding and neutralizing DENV, reaffirming that antibody epitope/paratope interactions occur independently of an antibody's $\mathrm{F}_{\mathrm{c}}$ domain.

\section{DENV-Reactive IgA Is Incapable of Mediating ADE}

Having demonstrated that the antigen binding and neutralization capacity of DENV-reactive monoclonal antibodies is negligibly impacted by the isotype of the construct, we endeavored to determine if the infection-enhancing capability of these antibodies was impacted by their isotype conversion. To this end, we utilized a K562-based ADE assay, wherein antibody/ DENV immune complexes were pre-formed and added to the $\mathrm{F}_{\mathrm{c}^{-}}$ receptor expressing K562 cell line to assess the ability of defined antibody complexes to enhance DENV infection.

The IgG versions of both VDB33 and VDB50 exhibited potent infection-enhancing activity in the K562 ADE assay, with both antibodies capable offacilitating DENV infection/enhancement in a dose-dependent fashion (Figures 2A-D). Consistent with their relative $\mathrm{EC}_{50} / \mathrm{IC}_{50}$ values, VDB33-IgG exhibited notably higher ADE activity than VDB50-IgG, but with the peak of ADE activity occurring at a similar antibody concentration. However, no infection enhancement was observed when the same assay was performed with either VDB33-IgA or VDB50-IgA (Figures 2A-D). This was despite the fact that these IgA isotype antibodies exhibit nearly identical virus binding and neutralization activity as their IgG counterparts, underlining the obligate role of an antibody's $\mathrm{F}_{\mathrm{c}}$ domain in determining the ADE potential of an antibody.

\section{DENV-Reactive IgA Antagonizes IgG- Mediated Enhancement of DENV Infection}

In light of the inability of VDB33-IgA and VDB50-IgA to facilitate ADE of DENV-3, we next endeavored to determine how DENVreactive IgG and IgA behave in a competitive setting. IgG and IgA antibodies are never found in isolation in a dengue immune individual, so determining how these antibodies function in a complex/poly-immune setting is critical for understanding their potential contribution to functional anti-DENV immunity.

To this end, we utilized the same K562 ADE assay as previously described, but used a fractional IgG/IgA replacement strategy wherein the total amount of antibody remained the same across the different titration schemes but the ratio of IgG to IgA was varied from 100:0 to 0:100. The fractional addition of DENV-reactive IgA significantly reduced the ADE activity observed in cultures containing either VDB33-IgG or VDB50-IgG (Figure 3). While both VDB33-IgA and VDB50-IgA were capable of antagonizing
IgG-mediated ADE of DENV-3, the highly avid yet non-enhancing VDB33-IgA antibody was capable of dramatically blunting IgGmediated ADE even when used at low fractional concentrations. Of note, the addition of DENV-reactive IgA to these ADE assays does not appear to shift the antibody dilution at which maximal ADE activity is observed for any of the cultures. Rather, the addition of DENV-reactive IgA reduces the magnitude of infection achieved at any given antibody dilution. These results are consistent with IgA actively antagonizing IgG mediated ADE by competing with DENV-reactive IgG for the same viral epitopes.

\section{DENV-Reactive IgA Antagonizes DENV- Immune Plasma Mediated Enhancement of DENV Infection}

A limitation of the analysis presented thus far is that all the monoclonal antibodies used in this analysis have the same antigen specificity; namely, the fusion loop of the DENV E protein. Therefore, it is unclear what impact-if any-DENVreactive IgA would have in the presence of a polyclonal IgG repertoire of divergent DENV antigen specificity. Therefore, we endeavored to determine how the presence of either VDB33-IgA or VDB50-IgA impacts the infection-enhancing potential of polyclonal/DENV-immune serum.

Plasma from DENV-immune donors was screened to identify samples with both high DENV-3 reactive IgG titers by ELISA as well as DENV-3 enhancing activity in the K562 ADE assay. Samples from four subjects were selected for additional analysis based on these criteria (Figure 4A, Supplementary Figures 3, 4). VDB33IgA or VDB50-IgA were then titrated into cultures containing this enhancing DENV-immune plasma to determine if IgA isotype monoclonal antibodies could antagonize polyclonal enhancement of DENV-3 infection.

Consistent with what was observed with IgG monoclonal antibodies, the addition of VDB33-IgA or VDB50-IgA significantly suppressed ADE-mediated K562 infection with DENV-3 (Figures 4B, C). The additional of DENV-reactive IgA in these assays suppressed ADE-mediated infection by $75 \%-90 \%$ in a dose-dependent fashion, a result consistent with the concept that IgG antibodies targeting the fusion loop of the DENV E protein are particularly amenable to facilitating ADE activity and are abundant in DENV-immune serum $(34,35)$. Notably, an equal amount of isotype control human serum IgA had no impact on the ADE activity, suggesting that the observed suppression via VDB33- and VDB50-IgA is a function of antigen binding rather than $\mathrm{F}_{\mathrm{c}}$-mediated (Supplementary Figure 5).These data also indicate that even modest concentrations of DENV-reactive IgA can significantly antagonize polyclonal IgG-mediated enhancement of DENV infection, signifying that the presence of DENV E reactive IgA (especially fusion loop reactive IgA) has the potential to significantly modulate DENV infection and associated immunopathogenesis. 
A

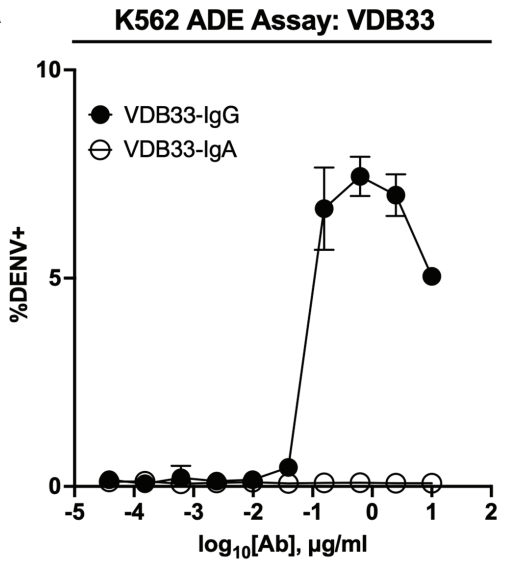

C

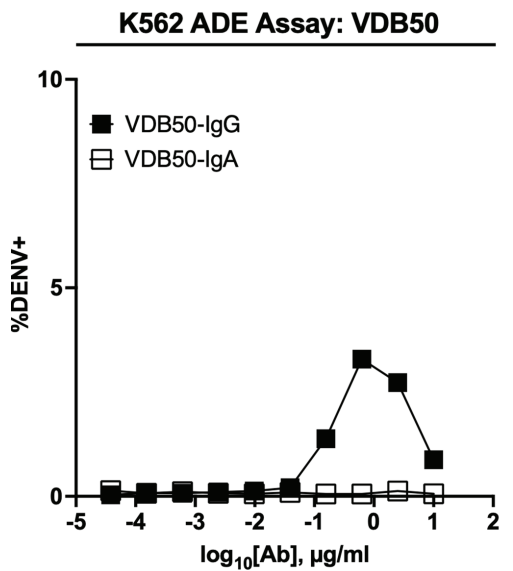

B

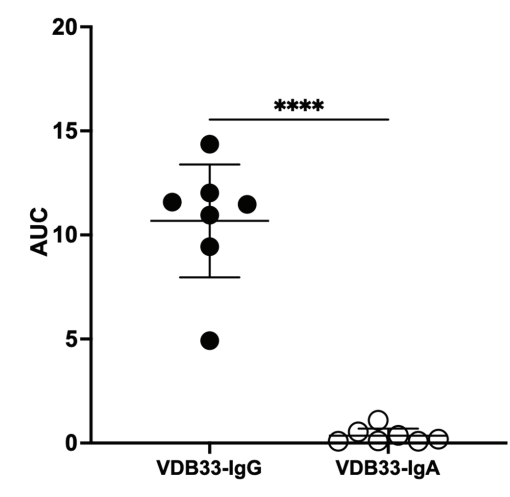

D

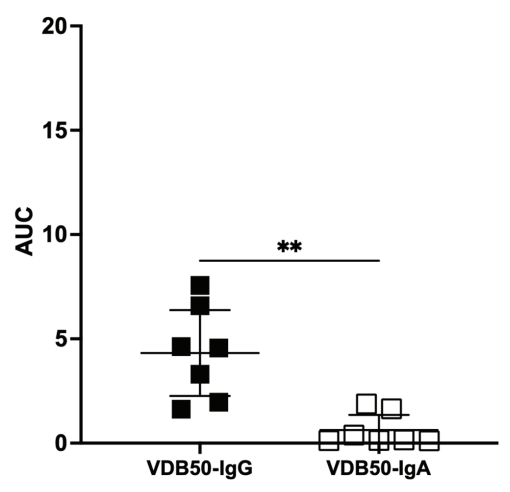

FIGURE 2 | ADE activity of DENV-reactive IgG and IgA isotype antibodies. (A) ADE activity of VDB33-IgG and VDB33-lgA against DENV-3 in K562 cells. (B) AUC values of 7 independent experimental replicates of DENV-3 ADE assay with VDB33-IgG and VDB33-IgA (C) ADE activity of VDB50-IgG and VDB50-IgA against DENV-3 in K562 cells. (D) AUC values of 7 independent replicates of DENV-3 ADE assay with VDB50-IgG and VDB50-IgA. Error bars $+/-$ SEM. ${ }^{* *} \mathrm{p}<0.01$, ${ }^{* \star \star *} \mathrm{p}<0.0001$, unpaired $t$ test. Data are representative of at least 7 independent experiments.

\section{DISCUSSION}

In this study we demonstrate that DENV-reactive IgA monoclonal antibodies can bind and neutralize DENV but are incapable of facilitating $\mathrm{ADE}$ of $\mathrm{DENV}$ infection in vitro. Furthermore, the presence of DENV-reactive IgA can significant blunt the DENVinfection enhancing activity of both DENV-reactive monoclonal IgG and polyclonal DENV-immune serum in a completive fashion. These results suggest an unappreciated role for DENV-reactive IgA during the humoral response to DENV infection and raise the potential that IgA could act as either a natural or therapeutic regulator of DENV dissemination and infection-attendant inflammation.

Although we have shown that DENV-reactive IgA is capable of disruption IgG mediated ADE, IgA may not be unique in this respect. Indeed, the depletion of IgM from flavivirus-immune serum has been shown to increase antibody-dependent enhancement of Zika virus infection of K562 cells, presumably by removing $\operatorname{IgM}$ as an antagonist of IgG-mediated infection enchantment (36). Accordingly, parallel lines of evidence suggest that the ability of DENV-reactive IgG to interact with FcyRs is linked to the infection-enhancing potential of DENV-immune serum and - by extension - the clinical severity of DENV infection. Polymorphisms in FcyRIIa - a component of the lowaffinity IgG receptor complex - have been associated with a decreased likelihood of becoming either symptomatically infected or progressing to severe dengue after DENV exposure (37-39). While the mechanism behind the phenomenon is hasn't been definitively established, it has been shown that at least some of these polymorphisms decrease the affinity of FcyRIIa for IgG (40). Furthermore, post-translational modifications of $\mathrm{IgG}$ antibodies have been shown to significant impact their affinity for Fc $\gamma \mathrm{R}$ complexes and to correlate with dengue severity (41). Most notably, the presence of high levels of afucosylated IgG- a posttranslational modification which increases the affinity of the IgG Fc domain for FcyRIIIa (42) - either before or after DENV infection has been associated with increased dengue severity (43-45). Finally, the presence of serum complement - such as C1q and C3 - can inhibit IgG mediated ADE both in vitro and in vivo, ostensibly by 
A

VDB33-IgG + VDB33-IgA
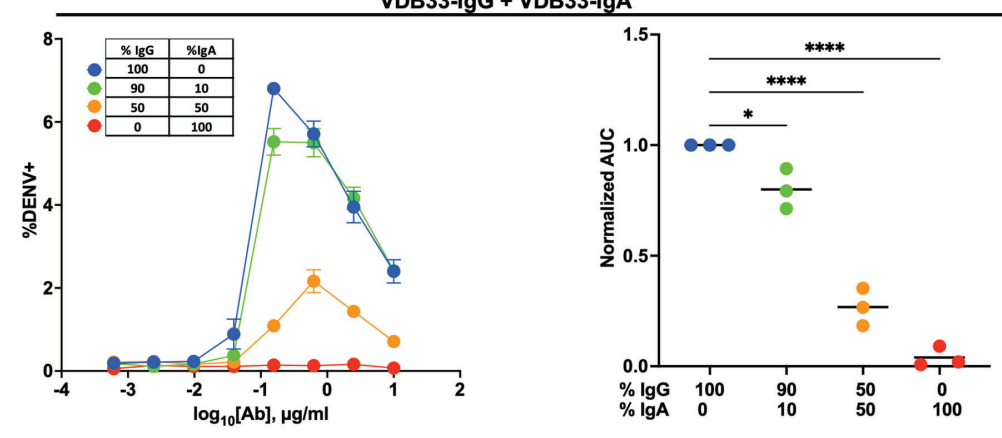

B

VDB33-IgG + VDB50--IgA
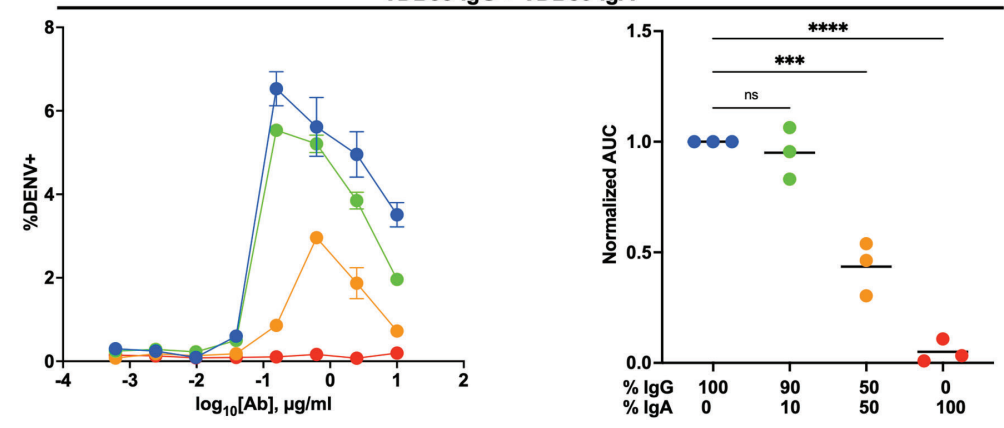

C

VDB50-IgG + VDB33-IgA
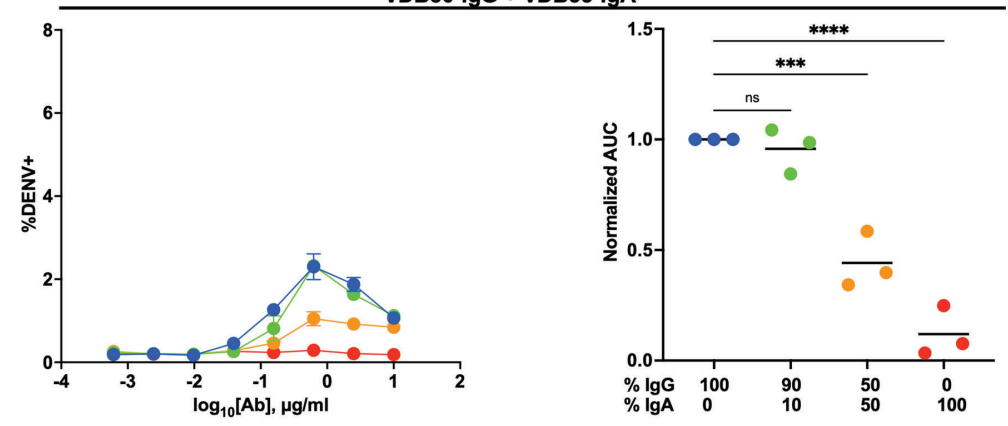

D

VDB50-IgG + VDB50-IgA
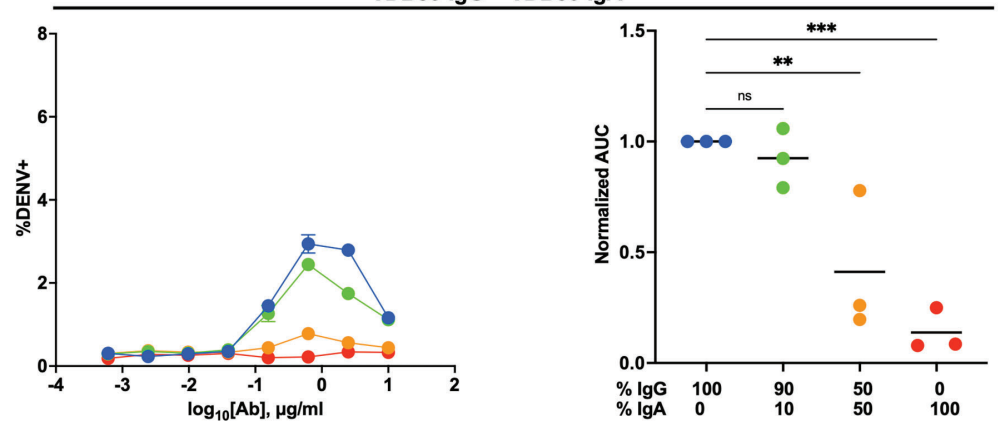

FIGURE 3 | Homotypic and heterotypic monoclonal IgA antagonizes lgG-mediated antibody-dependent enhancement. (A) DENV-3 ADE activity of VDB33-IgG when antagonized with VDB33-IgA. Total antibody concentration for each dilution point was held constant, with varying ratios of VDB33-IgG and VDB33-IgA as indicated. AUC of each ADE titration was calculated and normalized to that of the 100\% IgG condition. (B) DENV-3 ADE activity of VDB33-IgG when antagonized with VDB50-IgA. The AUC of each ADE titration was calculated and normalized to that of the 100\% VDB33-IgG condition. (C) DENV-3 ADE activity of VDB50-IgG when antagonized with VDB33-IgA. AUC of each ADE titration was calculated and normalized to that of the 100\% VDB50-IgG condition. (D) DENV-3 ADE activity of VDB50-IgG when antagonized with VDB50-IgA. AUC of each ADE titration was calculated and normalized to that of the 100\% VDB33-IgG condition. Blue $=100 \%$ $\lg \mathrm{G} / 0 \% \lg A$. Green $=90 \% \lg \mathrm{G} / 10 \% \lg A$. Orange $=50 \% \operatorname{lgG} / 50 \% \lg A$. Red $=0 \% \lg \mathrm{G} / 100 \% \lg \mathrm{A}$. "ns" denotes "non-significant" $(p>0.05) .{ }^{*} p<0.05,{ }^{* \star} p<0.01$, ${ }^{* \star *} \mathrm{p}<0.001,{ }^{* \star \star *} \mathrm{p}<0.00011$-way ANOVA with Dunnett correction for multiple comparisons. Data are representative of at least 4 independent experiments. 
A

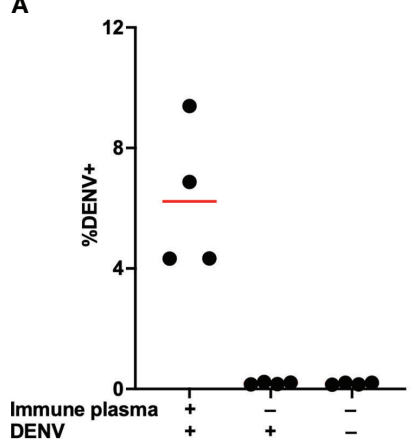

B

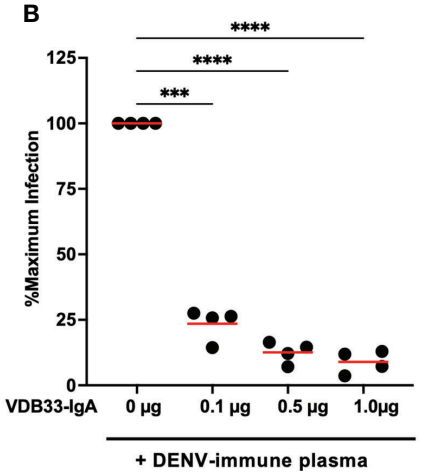

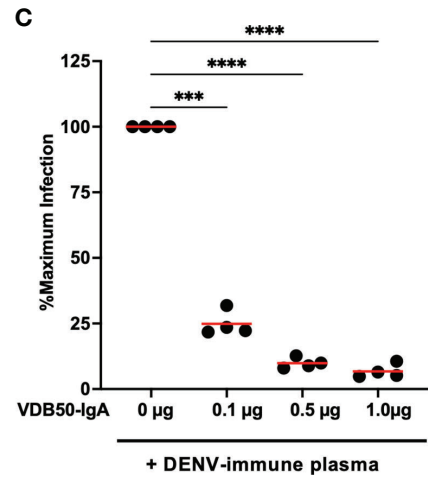

FIGURE 4 | Monoclonal IgA antagonizes ADE mediated by polyclonal DENV-immune plasma. (A) DENV immune plasma enhances DENV-3 infection of K562 cells. Each datapoint represents a unique plasma donor $(n=4)$. (B) VDB33-IgA antagonizes in vitro enhancement of DENV-3 infection mediated by polyclonal DENVimmune serum. Serum used at a 1:50 dilution for ADE assay, $n=4$ unique plasma donors. The percentage of DENV-positive cells was normalized to that observed in the plasma-only condition. (C) VDB50-IgA antagonizes in vitro enhancement of DENV-3 infection mediated by polyclonal DENV-immune serum. Serum used at a 1:50 dilution for $\mathrm{ADE}$ assay, $\mathrm{n}=4$ unique plasma donors. The percentage of DENV-positive cells was normalized to that observed in the plasma-only condition. ${ }^{* \star \star} \mathrm{p}<0.001,{ }^{\star \star \star \star} \mathrm{p}<0.00011$-way ANOVA with Dunnett correction for multiple comparisons. Data are representative of at least 4 independent experiments.

interfering with the ability of IgG Fc to interact with Fc $\gamma \mathrm{R}$ and/or forcing a complement-bound antibody into a configuration that is not amenable to fusion and viral entry (46-48).

While not found in nature, abolishing the ability of IgG antibodies to interact with $\mathrm{Fc} \gamma \mathrm{R}$ through genetic engineering the Fc portion of IgG (LALA mutation) also ablates many of the infection-enhancing properties of DENV-reactive IgG both in vitro (35) and in vivo $(49,50)$. Collectively, these this evidence underlines the importance of IgG/Fc $\gamma \mathrm{R}$ interactions the process of $\mathrm{ADE}$, and emphasize the potential diagnostic and therapeutic implications for factors that can disrupt this immunologic nexus.

The data presented herein demonstrate that DENV-reactive IgA is capable of antagonizing IgG mediated enhancement of DENV infection, yet it is still unclear what role this process plays in vivo during natural DENV infection. Several previous studies have noted that high levels of serum IgA are associated with more severe disease following secondary DENV infection (28-31). However, we and others have observed a higher frequency of IgA expressing plasmablasts following uncomplicated primary DENV infection than following severe secondary DENV infections $(32,33)$. A key takeaway from the analysis performed in this study is that the absolute concentration of a given DENV-reactive antibody isotype may be a incomplete indicator of the infection-enhancing potential of a serum sample. Severe dengue is accompanied by robust production of antibodies of all isotypes, so without additional context the absolute level of any given single antibody isotype may provide an incomplete or misleading impression of immunologic features associated with disease severity.

\section{MATERIALS AND METHODS}

\section{Viruses}

DENV-3 (strain CH53489) propagated in Vero cells were utilized for ELISA, FlowNT50, and ADE assays. Virus for
ELISA was purified by ultracentrifugation through a $30 \%$ sucrose solution and the virus pellet was resuspended in PBS.

Cell lines: Human K562 cells were maintained in IMDM supplemented with $10 \%$ FBS, penicillin, and streptomycin. U937-DC-SIGN cells were maintained in RPMI supplemented with $10 \%$ FBS, L-glutamine, penicillin, and streptomycin.

\section{Monoclonal Antibodies and Serum}

The variable regions from the heavy and light chains were codon optimized, synthesized in vitro and subcloned into a pcDNA3.4 vector containing the human IgG1 or IgA1 Fc region by a commercial partner (Genscript). Transfection grade plasmids were purified by maxiprep and transfected into a $293-6 \mathrm{E}$ expression system. Cells were grown in serum-free FreeStyle 293 Expression Medium (Thermo Fisher), and the cell supernatants collected on day 6 for antibody purification. Following centrifugation and filtration, the cell culture supernatant was loaded onto an affinity purification column, washed, eluted, and buffer exchanged to the final formulation buffer (PBS). Antibody lot purity was assessed by SDS-PAGE, and the final concentration determined by $280 \mathrm{~nm}$ absorption. The clonotype information for all monoclonal antibodies generated as part of this study is listed in Table 1. Dengue IgG antibody positive plasma was purchased from SeraCare. Donor ID and batch numbers are shown in Supplementary Table 1. Purified polyclonal IgA from human serum was purchased from a commercial source (Sigma, I4036-1mg).

\section{DENV- Capture ELISA}

Monoclonal antibody and plasma DENV-reactivity was assessed using a 4G2 DENV capture ELISA protocol. In short, 96 well NUNC MaxSorb flat-bottom plates were coated with $2 \mu \mathrm{g} / \mathrm{ml}$ flavivirus group-reactive mouse monoclonal antibody $4 \mathrm{G} 2$ (Envigo Bioproducts, Inc.) diluted in borate saline buffer. Plates were washed and blocked with $0.25 \%$ BSA $+1 \%$ Normal 
Goat Serum in PBS after overnight incubation. DENV-3 (strain CH53489) diluted in blocking buffer was captured for $2 \mathrm{hr}$, followed by extensive washing with PBS $+0.1 \%$ Tween 20 . Serially diluted monoclonal antibody or plasma samples were incubated for $1 \mathrm{hr}$ at RT on the captured virus, and DENVspecific antibody binding quantified using anti-human IgG HRP (Sigma-Aldrich, SAB3701362), anti-human IgA HRP (Biolegend, 411,002), or anti-human IgM HRP (SeraCare, 5220-0328). Secondary antibody binding was quantified using the TMB Microwell Peroxidase Substrate System (KPL, cat. \#5076-00) and Synergy HT plate reader (BioTek, Winooski, VT). Monoclonal antibody binding data were analyzed by nonlinear regression (One site total binding) to determine EC50 titers in GraphPad Prism 8 (GraphPad Software, La Jolla, CA). End-point titers of DENV-reactive plasma samples were determined as the reciprocal of the final dilution at which the optical density (OD) was greater than $2 \times$ of a control flavivirus naïve serum.

\section{Neutralization Assay}

Neutralizing titers of monoclonal antibodies and heatinactivated plasma were assessed using a flow cytometry-based neutralization assay in U937 cells expressing DC-SIGN as previously described $(51,52)$. Four-fold dilutions of antibody or sera were mixed with an equal volume of virus diluted to a concentration to achieve 10\%-15\% infection of U937-DC-SIGN cells in the absence of antibody. The antibody/virus mixture was incubated for $1 \mathrm{~h}$ at $37^{\circ} \mathrm{C}$, after which an equal volume of medium (RPMI-1640 supplemented with 10\% FBS, 1\% penicillin/streptomycin, 1\% l-glutamine $(200 \mathrm{mM})$ containing $5 \times 10^{4}$ U937-DC-SIGN cells was added to each well and incubated $18-20 \mathrm{hr}$ overnight in a $37^{\circ} \mathrm{C}, 5 \% \mathrm{CO} 2$, humidified incubator. Following overnight incubation, the cells were fixed with IC Fixation Buffer (Invitrogen, 00-82222-49), permeabilized using IC Permeabilization Buffer (Invitrogen, 00-8333-56) and immunostained with flavivirus group-reactive mouse monoclonal antibody 4G2 (Envigo Bioproducts, Inc.), and secondary polyclonal goat anti-mouse IgG PE-conjugated antibody (\#550589, BD Biosciences). The percentage of infected cells were quantified on a BD Accuri C6 Plus flow cytometer (BD Biosciences). Data were analyzed by nonlinear

\section{REFERENCES}

1. Gubler DJ. Aedes Aegypti and Aedes Aegypti-Borne Disease Control in the 1990s: Top Down or Bottom Up. Charles Franklin Craig Lecture. Am J Trop Med Hyg (1989) 40(6):571-8. doi: 10.4269/ajtmh.1989.40.571

2. Bhatt S, Gething PW, Brady OJ, Messina JP, Farlow AW, Moyes CL, et al. The Global Distribution and Burden of Dengue. Nature (2013) 496(7446):504-7. doi: $10.1038 /$ nature 12060

3. Shepard DS, Coudeville L, Halasa YA, Zambrano B, Dayan GH. Economic Impact of Dengue Illness in the Americas. Am J Trop Med Hyg (2011) 84 (2):200-7. doi: 10.4269/ajtmh.2011.10-0503

4. Guzman MG, Harris E. Dengue. Lancet (2015) 385(9966):453-65. doi: 10.1016/S0140-6736(14)60572-9

5. Sangkawibha N, Rojanasuphot S, Ahandrik S, Viriyapongse S, Jatanasen S, Salitul V, et al. Risk Factors in Dengue Shock Syndrome: A Prospective Epidemiologic Study in Rayong, Thailand. I. The 1980 Outbreak. Am J Epidemiol (1984) 120(5):653-69. doi: 10.1093/oxfordjournals.aje.a113932 regression to determine 50\% neutralization titers in GraphPad Prism 8 (GraphPad Software, La Jolla, CA).

\section{ADE Assay}

In vitro antibody-dependent enhancement (ADE) of DENV-3 infection was quantified as previously described $(32,53)$. Fourfold serial dilutions of antibody or heat-inactivated sera were incubated with virus (in sufficient amounts to infect $10 \%-15 \%$ of U937-DC-SIGN cells) at a $1: 1$ ratio for $1 \mathrm{~h}$ at $37^{\circ} \mathrm{C}$. This mixture was then added to a 96-well plate containing $5 \times 10^{4} \mathrm{~K} 562$ cells per well in duplicate. Cells were cultured for 18-20 hr overnight in a $37^{\circ} \mathrm{C}, 5 \% \mathrm{CO} 2$, humidified incubator. Processing and quantification continued as outlined in the FlowNT50 methods.

\section{Statistical Analysis}

All statistical analysis was performed using GraphPad Prism 8 Software (GraphPad Software, La Jolla, CA). A P-value $<0.05$ was considered significant.

\section{DATA AVAILABILITY STATEMENT}

The raw data supporting the conclusions of this article will be made available by the authors, without undue reservation.

\section{AUTHOR CONTRIBUTIONS}

ADW, AR, ST, TE, MM, JC, HF, GG, and ATW contributed to conception and design of the study. ADW and HSF performed analysis. ATW and ADW wrote the first draft of the manuscript. All authors contributed to manuscript revision, read, and approved the submitted version.

\section{SUPPLEMENTARY MATERIAL}

The Supplementary Material for this article can be found online at: https://www.frontiersin.org/articles/10.3389/fimmu.2021.777672/ full\#supplementary-material

6. Thein S, Aung MM, Shwe TN, Aye M, Zaw A, Aye K, et al. Risk Factors in Dengue Shock Syndrome. Am J Trop Med Hyg (1997) 56(5):566-72. doi: 10.4269/ajtmh.1997.56.566

7. Yoon IK, Srikiatkhachorn A, Hermann L, Buddhari D, Scott TW, Jarman RG, et al. Characteristics of Mild Dengue Virus Infection in Thai Children. Am J Trop Med Hyg (2013) 89(6):1081-7. doi: 10.4269/ajtmh.13-0424

8. Guzman MG, Alvarez M, Halstead SB. Secondary Infection as a Risk Factor for Dengue Hemorrhagic Fever/Dengue Shock Syndrome: An Historical Perspective and Role of Antibody-Dependent Enhancement of Infection. Arch Virol (2013) 158(7):1445-59. doi: 10.1007/s00705-0131645-3

9. Halstead SB. Dengue Antibody-Dependent Enhancement: Knowns and Unknowns. Microbiol Spectr (2014) 2(6):2-6. doi: 10.1128/microbiolspec.AID0022-2014

10. Diamond MS, Pierson TC. Molecular Insight Into Dengue Virus Pathogenesis and Its Implications for Disease Control. Cell (2015) 162(3):488-92. doi: 10.1016/j.cell.2015.07.005 
11. Halstead SB. Neutralization and Antibody-Dependent Enhancement of Dengue Viruses. Adv Virus Res (2003) 60:421-67. doi: 10.1016/S0065-3527 (03)60011-4

12. Kliks SC, Nimmanitya A, Nisalak A, Burke DS. Evidence That Maternal Dengue Antibodies are Important in the Development of Dengue Hemorrhagic Fever in Infants. Am J Trop Med Hyg (1988) 38(2):411-9. doi: 10.4269/ajtmh.1988.38.411

13. Chau TNB, Hieu NT, Anders KL, Wolbers M, Bich LL, Minh HLT, et al. Dengue Virus Infections and Maternal Antibody Decay in a Prospective Birth Cohort Study of Vietnamese Infants. J Infect Dis (2009) 200(12):1893-900. doi: $10.1086 / 648407$

14. Halstead SB, Lan NT, Myint TT, Shwe TN, Nisalak A, Kalyanarooj S, et al. Dengue Hemorrhagic Fever in Infants: Research Opportunities Ignored. Emerg Infect Dis (2002) 8(12):1474-9. doi: 10.3201/eid0812.020170

15. Goncalvez AP, Engle RE, St. Claire M, Purcell RH, Lai CJ. Monoclonal Antibody-Mediated Enhancement of Dengue Virus Infection In Vitro and In Vivo and Strategies for Prevention. Proc Natl Acad Sci USA (2007) 104 (22):9422-7. doi: 10.1073/pnas.0703498104

16. Zellweger RM, Prestwood TR, Shresta S. Enhanced Infection of Liver Sinusoidal Endothelial Cells in a Mouse Model of Antibody-Induced Severe Dengue Disease. Cell Host Microbe (2010) 7(2):128-39. doi: 10.1016/j.chom.2010.01.004

17. Guzman MG, Vazquez S. The Complexity of Antibody-Dependent Enhancement of Dengue Virus Infection. Viruses (2010) 2(12):2649-62. doi: $10.3390 / \mathrm{v} 2122649$

18. Katzelnick LC, Gresh L, Halloran ME, Mercado JC, Kuan G, Gordon A, et al. Antibody-Dependent Enhancement of Severe Dengue Disease in Humans. Science (2017) 358(6365):929-32. doi: 10.1126/science.an6836

19. McCracken MK, Kuklis CH, Kannadka CB, Barvir DA, Sanborn MA, Waickman AT, et al. Enhanced Dengue Vaccine Virus Replication and Neutralizing Antibody Responses in Immune Primed Rhesus Macaques. NPJ Vaccines (2021) 6(1):77. doi: 10.1038/s41541-021-00339-y

20. Thomas SJ, Endy TP. Critical Issues in Dengue Vaccine Development. Curr Opin Infect Dis (2011) 24(5):442-50. doi: 10.1097/QCO.0b013e32834a1b0b

21. Hadinegoro SR, Arrendono-Garcia JL, Capeding MR, Deseda C, Chotpitayasunondh T, Dietze R, et al. Efficacy and Long-Term Safety of a Dengue Vaccine in Regions of Endemic Disease. N Engl J Med (2015) 373 (13):1195-206. doi: 10.1056/NEJMoa1506223

22. Sridhar S, Luedtke A, Langevin E, Zhu M, Bonaparte M, Machabert T, et al. Effect of Dengue Serostatus on Dengue Vaccine Safety and Efficacy. N Engl J Med (2018) 379(4):327-40. doi: 10.1056/NEJMoa1800820

23. Ferguson NM, Rodriguez-Barraquer I, Dorigatti I, Mier-Y-Teran-Romero L, Laydon DJ, Cummings DAT. Benefits and Risks of the Sanofi-Pasteur Dengue Vaccine: Modeling Optimal Deployment. Science (2016) 353(6303):1033-6. doi: 10.1126/science.aaf9590

24. Guzman MG, Gubler DJ, Izquierdo A, Martinez E, Halstead SB. Dengue Infection. Nat Rev Dis Primers (2016) 2:16055. doi: 10.1038/nrdp.2016.55

25. St John AL, Rathore APS. Adaptive Immune Responses to Primary and Secondary Dengue Virus Infections. Nat Rev Immunol (2019) 19(4):218-30. doi: 10.1038/s41577-019-0123-x

26. Macpherson AJ, McCoy KD, Johansen F-E, Brandtzaeg P. The Immune Geography of IgA Induction and Function. Mucosal Immunol (2008) 1 (1):11-22. doi: $10.1038 / \mathrm{mi} .2007 .6$

27. Alagarasu K, Walimbe AM, Jadhav SM, Deoshatwar AR. A Meta-Analysis of the Diagnostic Accuracy of Dengue Virus-Specific IgA Antibody-Based Tests for Detection of Dengue Infection. Epidemiol Infect (2016) 144(4):876-86. doi: 10.1017/S0950268815001922

28. Koraka P, Suharti C, Setiati TE, Mairuhu AT, Van Gorp E, Hack CE, et al. Kinetics of Dengue Virus-Specific Serum Immunoglobulin Classes and Subclasses Correlate With Clinical Outcome of Infection. J Clin Microbiol (2001) 39(12):4332-8. doi: 10.1128/JCM.39.12.4332-4338.2001

29. Vazquez S, Lozano C, Perez AB, Castellanos Y, Ruiz D, Calzada N, et al. Dengue Specific Immunoglobulins M, A, and E in Primary and Secondary Dengue 4 Infected Salvadorian Children. J Med Virol (2014) 86(9):1576-83. doi: $10.1002 / j m v .23833$

30. Bachal R, Alagarasu RK, Singh A, Salunke A, Shah P, Cecilia D. Higher Levels of Dengue-Virus-Specific IgG and IgA During Pre-Defervescence Associated With Primary Dengue Hemorrhagic Fever. Arch Virol (2015) 160(10):243543. doi: $10.1007 / \mathrm{s} 00705-015-2519-7$
31. Zhao H, Qiu S, Hong WX, Song KY, Wang J, Yang HQ, et al. Dengue Specific Immunoglobulin A Antibody is Present in Urine and Associated With Disease Severity. Sci Rep (2016) 6:27298. doi: 10.1038/srep27298

32. Waickman AT, Gromowski GD, Rutvisuttinunt W, Li T, Siegfried H, Victor K, et al. Transcriptional and Clonal Characterization of B Cell Plasmablast Diversity Following Primary and Secondary Natural DENV Infection. EBioMedicine (2020) 54:102733. doi: 10.1016/j.ebiom.2020.102733

33. Rouers A, Appanna R, Chevrier M, Lum J, Lau MC, Tan L, et al. CD27(hi) CD38(hi) Plasmablasts are Activated B Cells of Mixed Origin With Distinct Function. iScience (2021) 24(5):102482. doi: 10.1016/j.isci.2021.102482

34. Rodenhuis-Zybert IA, Moesker B, Voorham JM, Ende-Meteselaar H, Diamond MS, Wilschut J, et al. A Fusion-Loop Antibody Enhances the Infectious Properties of Immature Flavivirus Particles. J Virol (2011) 85 (22):11800-8. doi: 10.1128/JVI.05237-11

35. Beltramello M, Williams KL, Simmons CP, Macagno A, Simonelli L, Quyen NTH, et al. The Human Immune Response to Dengue Virus is Dominated by Highly Cross-Reactive Antibodies Endowed With Neutralizing and Enhancing Activity. Cell Host Microbe (2010) 8(3):271-83. doi: 10.1016/ j.chom.2010.08.007

36. Malafa S, Medits I, Aberle JH, Aberle SW, Halswanter D, Tsouchnikas SG, et al. Impact of Flavivirus Vaccine-Induced Immunity on Primary Zika Virus Antibody Response in Humans. PLoS Negl Trop Dis (2020) 14(2):e0008034. doi: 10.1371/journal.pntd.0008034

37. Loke H, Bethell D, Xuan C, Phuong CXT, Day N, White N, et al. Susceptibility to Dengue Hemorrhagic Fever in Vietnam: Evidence of an Association With Variation in the Vitamin D Receptor and Fc Gamma Receptor IIa Genes. Am J Trop Med Hyg (2002) 67(1):102-6. doi: 10.4269/ajtmh.2002.67.102

38. Mohsin SN, Mahmood S, Amar A, Ghafoor F, Raza SM, Saleem M. Association of FcgammaRIIa Polymorphism With Clinical Outcome of Dengue Infection: First Insight From Pakistan. Am J Trop Med Hyg (2015) 93(4):691-6. doi: 10.4269/ajtmh.15-0199

39. García G, Sierra B, Pérez AB, Aguirre E, Rosado I, Gonzalez N, et al. Asymptomatic Dengue Infection in a Cuban Population Confirms the Protective Role of the RR Variant of the FcgammaRIIa Polymorphism. Am J Trop Med Hyg (2010) 82(6):1153-6. doi: 10.4269/ajtmh.2010.09-0353

40. Warmerdam PA, van de Winkel JG, Vlug A, Westerdaal NA, Capel PJ. A Single Amino Acid in the Second Ig-Like Domain of the Human Fc Gamma Receptor II is Critical for Human IgG2 Binding. J Immunol (1991) 147(4):1338-43.

41. Bournazos S, Gupta A, Ravetch JV. The Role of IgG Fc Receptors in AntibodyDependent Enhancement. Nat Rev Immunol (2020) 20(10):633-43. doi: 10.1038/s41577-020-00410-0

42. Herter S, Herting F, Mundigl O, Waldhauer I, Weinzierl T, Fauit T, et al. Preclinical Activity of the Type II CD20 Antibody GA101 (Obinutuzumab) Compared With Rituximab and Ofatumumab In Vitro and in Xenograft Models. Mol Cancer Ther (2013) 12(10):2031-42. doi: 10.1158/1535-7163.MCT-12-1182

43. Bournazos S, Vo HTM, Duong V, Auerswald H, Ly S, Sakuntabhai A, et al. Antibody Fucosylation Predicts Disease Severity in Secondary Dengue Infection. Science (2021) 372(6546):1102-5. doi: 10.1126/science.abc7303

44. Wang TT, Sewatanon J, Memoli MJ, Wrammert J, Bourznos S, Bhaumik SK, et al. IgG Antibodies to Dengue Enhanced for FcgammaRIIIA Binding Determine Disease Severity. Science (2017) 355(6323):395-8. doi: 10.1126/science.aai8128

45. Thulin NK, Brewer CR, Sherwood R, Bournazos S, Edwards G, Ramadoss NS, et al. Maternal Anti-Dengue IgG Fucosylation Predicts Susceptibility to Dengue Disease in Infants. Cell Rep (2020) 31(6):107642. doi: 10.1016/j.celrep.2020.107642

46. Mehlhop E, Ansarah-Sobrinho C, Johnson S, Engle M, Fremont DH, Pierson TC, et al. Complement Protein C1q Inhibits Antibody-Dependent Enhancement of Flavivirus Infection in an IgG Subclass-Specific Manner. Cell Host Microbe (2007) 2(6):417-26. doi: 10.1016/j.chom.2007.09.015

47. Yamanaka A, Kosugi S, Konishi E. Infection-Enhancing and -Neutralizing Activities of Mouse Monoclonal Antibodies Against Dengue Type 2 and 4 Viruses are Controlled by Complement Levels. J Virol (2008) 82(2):927-37. doi: 10.1128/JVI.00992-07

48. Byrne AB, Talarico LB. Role of the Complement System in AntibodyDependent Enhancement of Flavivirus Infections. Int J Infect Dis (2021) 103:404-11. doi: 10.1016/j.ijid.2020.12.039

49. Xu M, Zuest R, Velumani S, Tukijan F, Toh YX, Appanna R, et al. A Potent Neutralizing Antibody With Therapeutic Potential Against All Four Serotypes of Dengue Virus. NPJ Vaccines (2017) 2:2. doi: 10.1038/s41541-016-0003-3 
50. Balsitis SJ, Williams KL, Lachica R, Flores D, Kyle JL, Melhop E, et al. Lethal Antibody Enhancement of Dengue Disease in Mice is Prevented by $\mathrm{Fc}$ Modification. PLoS Pathog (2010) 6(2):e1000790. doi: 10.1371/ journal.ppat.1000790

51. Kraus AA, Messer W, Haymore LB, de Silva AM. Comparison of Plaque- and Flow Cytometry-Based Methods for Measuring Dengue Virus Neutralization. J Clin Microbiol (2007) 45(11):3777-80. doi: 10.1128/JCM.00827-07

52. de Alwis R, Smith SA, Olivarez NP, Messer WN, Huyng JP, Wahala WMP, et al. Identification of Human Neutralizing Antibodies That Bind to Complex Epitopes on Dengue Virions. Proc Natl Acad Sci USA (2012) 109(19):7439-44. doi: 10.1073/pnas.1200566109

53. McCracken MK, Gromowski MGD, Friberg HL, Lin X, Abbink P, De La Barrera R, et al. Impact of Prior Flavivirus Immunity on Zika Virus Infection in Rhesus Macaques. PLoS Pathog (2017) 13(8):e1006487. doi: 10.1371/ journal.ppat.1006487

Author Disclaimer: The opinions or assertions contained herein are the private views of the authors and are not to be construed as reflecting the official views of the US Army or the US Department of Defense. Material has been reviewed by the Walter Reed Army Institute of Research. There is no objection to its presentation and/or publication.
Conflict of Interest: Authors ADW, MM, JC, HF, GG, and ATW are co-inventors on the provisional patent "IgA monoclonal antibodies as a prophylactic and therapeutic treatment for acute flavivirus infection".

The remaining authors declare that the research was conducted in the absence of any commercial or financial relationships that could be construed as a potential conflict of interest.

Publisher's Note: All claims expressed in this article are solely those of the authors and do not necessarily represent those of their affiliated organizations, or those of the publisher, the editors and the reviewers. Any product that may be evaluated in this article, or claim that may be made by its manufacturer, is not guaranteed or endorsed by the publisher.

Copyright (C) 2021 Wegman, Fang, Rothman, Thomas, Endy, McCracken, Currier, Friberg, Gromowski and Waickman. This is an open-access article distributed under the terms of the Creative Commons Attribution License (CC BY). The use, distribution or reproduction in other forums is permitted, provided the original author(s) and the copyright owner(s) are credited and that the original publication in this journal is cited, in accordance with accepted academic practice. No use, distribution or reproduction is permitted which does not comply with these terms. 